\title{
Algorithm for positive realization of transfer functions
}

Béla Nagy, Máté Matolcsi

Béla Nagy is Professor at the Mathematics Department of Technical University, Budapest. E-mail: bnagy@math.bme.hu

Máté Matolcsi is a research assistant in Applied Mathematics at the Alfred Renyi Institute, Budapest. E-mail: matomate@renyi.hu 


\begin{abstract}
The aim of this brief is to present a finite-step algorithm for the positive realization of a rational transfer function $H(z)$. In comparision with previously described algorithms we emphasize that we $d o$ not make an a priori assumption on (but, instead, include a finite step procedure for checking) the nonnegativity of the impulse response sequence of $H(z)$. For primitive transfer functions a new method for reducing the pole order of the dominant pole is also proposed.
\end{abstract}

\title{
Keywords
}

Positive linear systems, impulse response sequence, positive realization.

\section{INTRODUCTION}

The positive realization problem of a given rational transfer function $H(z)$ of a discrete time-invariant linear system is to find a triple $A \in \mathbb{R}_{+}^{N \times N}, b \in \mathbb{R}_{+}^{N}, c \in \mathbb{R}_{+}^{N}$ (with nonnegative entries) such that $H(z)=c^{T}(z I-A)^{-1} b$ holds. The nonnegativity restriction on the entries of $A, b, c$ reflect physical constraints in applications. Such positive systems appear for example in modelling bio-systems, chemical reaction systems, and socio-economic systems, as described in detail in the monograph by Farina and Rinaldi [5]. A recent application of positive systems in the construction of CRN's (Charge Routing Networks) was given by Benvenuti, Farina and Anderson [2].

In [9] Ohta, Maeda and Kodama reduced the problem of positive realizability of $H(z)$ to finding an appropriate convex polyhedral cone in the room sandwiched by the reachability and observability cones in the state space of an arbitrary minimal realization of $H(z)$. However, the problem of constructing such a polyhedral cone turned out to be highly nontrivial (a characterization of all such cones is still lacking). In [1] Anderson, Deistler, Farina and Benvenuti proved that such a cone is always possible to construct if $H(z)$ is a primitive transfer function with nonnegative impulse response. Finally, the case of nonprimitive transfer functions $H(z)$ with nonegative impulse response was settled by Farina in [4] by the method of downsampling the impulse response of $H(z)$ (see also Kitano and Maeda [8], and Förster and Nagy [6]). A common feature of the results mentioned above is that the impulse response of $H(z)$ is assumed to be nonnegative. However, it has not been shown so far how one can check, in finite steps, the nonnegativity of the (clearly infinite) impulse response sequence corresponding to $H(z)$ (this open problem was raised in [1]). 
In the course of this brief we aim to supplement the theory of positive realizability by tackling this problem in Section 2. In Section 3 we propose a new method of constructing a positive realization of a primitive transfer function with multiple dominant pole. In Section 4 we illustrate our results by an example.

\section{The NONNEGAtivity OF The IMPUlSE RESPONSE SEQUENCE}

Let

$$
H(z)=\frac{p_{1} z^{n-1}+\ldots+p_{n}}{z^{n}+q_{1} z^{n-1}+\ldots+q_{n}}=\sum_{j=1}^{r} \sum_{i=1}^{n_{j}} \frac{c_{j}^{(i)}}{\left(z-\lambda_{j}\right)^{i}}
$$

be a strictly proper rational transfer function, where $\lambda_{1}$ denotes the nonnegative pole of $H(z)$ with greatest modulus. Note that the coefficients $p_{j}$ and $q_{j}$ are assumed to be real, but the poles $\lambda_{j}(j \neq 1)$ can be complex. We will use the notation $h_{k}(k=1,2,3 \ldots)$ for the impulse response sequence of $H(z)$, i.e. $H(z)=\sum_{k=1}^{\infty} h_{k} z^{-k}$. A minimal realization of $H(z)$ will be denoted by $(g, F, h)$. We will describe the structure of our algorithm in several steps. The first two steps are standard in the theory of positive realizations (cf. [1] and [4]), but we include them for completeness and convenience.

Step 1. It is well-known (cf. [1]) that a necessary condition for the existence of a positive realization of $H(z)$ is that $\lambda_{1}$ be a dominant pole of $H(z)$, i.e. the modulus of no pole exceed $\lambda_{1}$. Therefore, if there is no nonnegative pole of $H(z)$, or $\lambda_{1}$ is not dominant, we can conclude that $H(z)$ does not have positive realizations. Assume that $\lambda_{1}$ is dominant. If $\lambda_{1}=0$, then the realization problem is trivial, and if $\lambda_{1}>0$, then it is also well-known that we may (and will) assume without loss of generality that $\lambda_{1}=1$ (cf. [1]).

Step 2. If $H(z)$ is not primitive (i.e. $H(z)$ has dominant poles other than $\lambda_{1}=1$ ), then a necessary condition for the existence of a positive realization is that the dominant poles of $H(z)$ be cyclic (see [1]), i.e. there exist $p \in \mathbb{N}$ such that all the dominant poles of $H(z)$ satisfy the equation $z^{p}=1$. If the dominant poles are not cyclic, then we conclude that there is no positive realization of $H(z)$. If the dominant poles are cyclic with index $p$ (the smallest of the $p$ 's above), then the necessary and sufficient condition for positive realizability of $H(z)$ is that all the "downsampled" transfer functions $H_{(j)}(z):=g(z I-$ $\left.F^{p}\right)^{-1} F^{j} h$ (for $j=0,1, \ldots, p-1$ ) be positively realizable (see [4] and [8]). Notice that $H(z)=\sum_{j=0}^{p-1} z^{p-1-j} H_{(j)}\left(z^{p}\right)$. 
If some of the functions $H_{(j)}(z)$ are not primitive, then we apply the downsampling step again to these functions (provided that they are cyclic), and, following the algorithm of [4] (cf. also [8]), we arrive (after a finite number of steps) at a decomposition of the form

$$
H(z)=\sum_{s} z^{\beta_{s}} H_{(s)}\left(z^{\alpha_{s}}\right)
$$

where $0 \leq \beta_{s}<\alpha_{s}$, and all the functions $H_{(s)}(z)$ are either primitive or not cyclic. If for any $s$ the function $H_{(s)}(z)$ is not cyclic, then we conclude that $H(z)$ does not have positive realizations (cf. [4]). Assume therefore that all $H_{(s)}(z)$ are primitive. In this case, $H(z)$ is positive realizable if and only if the impulse response of $H(z)$ is nonnegative (cf. [4]).

Step 3. In this step we give an upper estimate on the finite number of the terms of the impulse response sequence $h_{k}$ of $H(z)$ whose nonnegativity we need to check in order to conclude that the whole impulse response sequence is nonnegative.

Instead of checking the impulse response of $H(z)$ directly, we take the decomposition $H(z)=\sum_{s} z^{\beta_{s}} H_{(s)}\left(z^{\alpha_{s}}\right)$ of Step 2, and check the impulse response of each $H_{(s)}(z)$. If the impulse response of each $H_{(s)}(z)$ is non-negative, then clearly so is the impulse response of $H(z)$. The advantage of this method is that all the functions $H_{(s)}(z)$ are primitive.

For the sake of simplicity we will still use the notation $H(z)$ instead of $H_{(s)}(z)$, but we will assume that $H(z)$ is primitive.

We shall use several times the following observation: if a transfer function $H(z)$ has the form $H(z)=\sum_{k=1}^{n} \frac{e_{k}}{(z-s)^{k}}$, then the impulse response sequence is

$$
h_{m}=\sum_{k=1}^{n}\left(\begin{array}{c}
m-1 \\
k-1
\end{array}\right) s^{m-k} e_{k} \quad m=1,2,3, \ldots,
$$

(with the convention that for $\alpha<\beta$ we define $\left(\begin{array}{l}\alpha \\ \beta\end{array}\right):=0$, and $\left(\begin{array}{l}0 \\ 0\end{array}\right):=1$ and $0^{0}:=1$ ). This can be proved by using for each $k=1,2, \ldots n$ and $|z|>|s|$ the formula $\frac{1}{(z-s)^{k}}=$ $\left(\frac{1}{z}\right)^{k}\left[1+\frac{s}{z}+\left(\frac{s}{z}\right)^{2}+\left(\frac{s}{z}\right)^{3}+\ldots\right]^{k}$. Since we have $\sum_{k=1}^{n} \frac{e_{k}}{(z-s)^{k}}=H(z)=\sum_{m=1}^{\infty} \frac{h_{m}}{z^{m}}$, comparing coefficients yields the stated formula.

Recall now that for our transfer function $H(z)$ the only dominant pole is $\lambda_{1}=1$. If $c_{1}^{\left(n_{1}\right)}<0$, then it is clear (see the explicit formula for $h_{k, 1}$ and the estimates on $h_{k}$ below) that for large $k$ we have $h_{k}<0$, hence there exists no positive realization of $H(z)$. Therefore we can assume that $c_{1}^{\left(n_{1}\right)}>0$, and, without loss of generality, that $c_{1}^{\left(n_{1}\right)}=1$. 
We use the notation $h_{k, j}^{(i)}$ for the impulse response sequence corresponding to the function $\frac{c_{j}^{(i)}}{\left(z-\lambda_{j}\right)^{i}}$, and we let $h_{k, j}:=\sum_{i=1}^{n_{j}} h_{k, j}^{(i)}$. Hence we shall have $h_{k}=\sum_{j=1}^{r} h_{k, j}$. The idea behind the forthcoming calculations is that $h_{k, 1}$ turns out to be 'dominant' in the long term behaviour of $h_{k}$, and $h_{k, 1}^{\left(n_{1}\right)}$ will be 'dominant' in the long term behaviour of $h_{k, 1}$.

First we find an index $N_{0}$ such that $h_{k, 1} \geq 1$ for all $k \geq N_{0}$. If $n_{1}=1$, then $h_{k, 1}=1$ for all $k \geq 1$, therefore we can take $N_{0}=1$. Assume $n_{1}>1$. Since we have $\lambda_{1}=1$ and $c_{1}^{\left(n_{1}\right)}=1$, it follows from (3) that

$$
\begin{aligned}
& h_{k, 1}=\left(\begin{array}{c}
k-1 \\
n_{1}-1
\end{array}\right)+c_{1}^{\left(n_{1}-1\right)}\left(\begin{array}{c}
k-1 \\
n_{1}-2
\end{array}\right)+\ldots+c_{1}^{(2)}\left(\begin{array}{c}
k-1 \\
1
\end{array}\right)+c_{1}^{(1)}= \\
& 1+\left[\left(\begin{array}{c}
k-1 \\
n_{1}-1
\end{array}\right)+c_{1}^{\left(n_{1}-1\right)}\left(\begin{array}{c}
k-1 \\
n_{1}-2
\end{array}\right)+\ldots+c_{1}^{(2)}\left(\begin{array}{c}
k-1 \\
1
\end{array}\right)+c_{1}^{(1)}-1\right] .
\end{aligned}
$$

Let $C:=\max \left\{\left|c_{1}^{\left(n_{1}-1\right)}\right|, \ldots,\left|c_{1}^{(2)}\right|,\left|c_{1}^{(1)}\right|+1\right\}$, and assume that $k \geq\left(n_{1} C+1\right)\left(n_{1}-1\right)=: N_{0}$. (Note that $N_{0} \geq 3 n_{1}-3 \geq 2 n_{1}-1$, so the finite sequence of the binomial coefficients $\left(\begin{array}{c}k-1 \\ n_{1}-1\end{array}\right),\left(\begin{array}{c}k-1 \\ n_{1}-2\end{array}\right), \ldots,\left(\begin{array}{c}k-1 \\ 0\end{array}\right)$ is strictly monotonically decreasing.) For $k$ this large we have $\frac{k-n_{1}+1}{n_{1}-1} \geq n_{1} C$ and this means that $\left(\begin{array}{c}k-1 \\ n_{1}-1\end{array}\right) /\left(\begin{array}{c}k-1 \\ n_{1}-2\end{array}\right) \geq n_{1} C$. Hence for any $2 \leq j \leq n_{1}$ we have $\left(\begin{array}{c}k-1 \\ n_{1}-1\end{array}\right) /\left(\begin{array}{c}k-1 \\ n_{1}-j\end{array}\right) \geq n_{1} C$. This means that

$$
\begin{gathered}
h_{k, 1}=1+\left[\left(\begin{array}{c}
k-1 \\
n_{1}-1
\end{array}\right)+c_{1}^{\left(n_{1}-1\right)}\left(\begin{array}{c}
k-1 \\
n_{1}-2
\end{array}\right)+\ldots+c_{1}^{(2)}\left(\begin{array}{c}
k-1 \\
1
\end{array}\right)+c_{1}^{(1)}-1\right] \geq \\
1+\left(\begin{array}{c}
k-1 \\
n_{1}-1
\end{array}\right)-C \sum_{i=1}^{n_{1}-1}\left(\begin{array}{c}
k-1 \\
i-1
\end{array}\right) \geq 1
\end{gathered}
$$

as desired.

Next we find an index $M_{0}$ such that $\sum_{j=2}^{r}\left|h_{k, j}\right| \leq 1$ for all $k>M_{0}$. It follows from (3) that $h_{k, j}=\sum_{i=1}^{n_{j}} h_{k, j}^{(i)}=\sum_{i=1}^{n_{j}} c_{j}^{(i)} \lambda_{j}^{k-i}\left(\begin{array}{c}k-1 \\ i-1\end{array}\right)$. Therefore

$$
\left|h_{k, j}\right| \leq \sum_{i=1}^{n_{j}}\left|h_{k, j}^{(i)}\right| \leq \sum_{i=1}^{n_{j}}\left|c_{j}^{(i)}\right|\left|\lambda_{j}\right|^{k-i}\left(\begin{array}{c}
k-1 \\
i-1
\end{array}\right) .
$$

Now, there are altogether $N_{1}:=\sum_{j=2}^{r} n_{j}$ coefficients of type $h_{k, j}^{(i)}$ so it is enough to ensure that the modulus of each of them is not greater than $1 / N_{1}$. That is, we want $\left|c_{j}^{(i)}\right|\left|\lambda_{j}\right|^{k-i}\left(\begin{array}{c}k-1 \\ i-1\end{array}\right) \leq \frac{1}{N_{1}}$ to hold. If $\lambda_{j}=0$, then this is obviously true for $k \geq i+1$. Assume $\lambda_{j} \neq 0$. To simplify forthcoming calculations we use the notation $\rho:=\max \left\{\left|\lambda_{j}\right|: j=\right.$ $2,3, \ldots, r\}, \gamma:=\max \left\{\left|c_{j}^{(i)}\right|: j=2,3, \ldots, r ; i=1,2, \ldots, n_{j}\right\}$ and $\eta:=\max \left\{n_{j}: j=\right.$ $2,3, \ldots, r\}$. The desired inequality $\left|c_{j}^{(i)}\right|\left|\lambda_{j}\right|^{k-i}\left(\begin{array}{c}k-1 \\ i-1\end{array}\right) \leq \frac{1}{N_{1}}$ is implied by $\gamma \rho^{k-i}\left(\begin{array}{c}k-1 \\ i-1\end{array}\right) \leq \frac{1}{N_{1}}$, which is equivalent to $\rho^{k / 2} \rho^{k / 2}\left(\begin{array}{c}k-1 \\ i-1\end{array}\right) \leq \frac{\rho^{i}}{N_{1} \gamma}$. It is easy to check that for fixed $i$ the value of $\rho^{k / 2}\left(\begin{array}{c}k-1 \\ i-1\end{array}\right)$ is monotonically decreasing (in $k$ ) for $k \geq \frac{i-1}{1-\rho^{1 / 2}}+1=: N^{(i)}$. 
We use the notation $C^{(i)}:=\rho^{\left(N^{(i)} / 2\right)}\left(\begin{array}{c}N^{(i)}-1 \\ i-1\end{array}\right)$. If $k \geq N^{(i)}$, then it is sufficient that

$$
\left(\rho^{1 / 2}\right)^{k} \leq \frac{\rho^{i}}{N_{1} \gamma C^{(i)}}=: K^{(i)}
$$

therefore we can take

$$
M_{0}:=\max \left\{\eta, N^{(i)}, \log _{\rho^{1 / 2}} K^{(i)}, i=1,2, \ldots \eta\right\}
$$

(Note that we include $\eta$ because we must not forget about the possible pole at 0 .)

This means that if $k>\max \left\{N_{0}, M_{0}\right\}$, then $h_{k} \geq h_{k, 1}-\sum_{j=2}^{r}\left|h_{k, j}\right| \geq 0$, therefore it is definitely enough to check the nonnegativity of the first $K_{0}:=\max \left\{N_{0}, M_{0}\right\}$ terms of the impulse response sequence. We remark that the calculations above show that it is sufficient to know an upper bound on the values of $\rho$ and $\gamma$ instead of the exact values. This means that it is enough to determine the approximate locations of the poles, and the approximate values of the partial fraction coefficients, and Step 3 can already be applied.

\section{A NONNEGATIVE REALIZATION}

Step 4. In order to construct a positive realization of $H(z)$ it is sufficient to find a positive realization for each $H_{(s)}(z)$ in the decomposition (2), and then apply the method of [4] or [8]. As all $H_{(s)}(z)$ are primitive, we could find a positive realization of $H_{(s)}(z)$ by applying the results of [1] directly. However, in this Step we propose a method for reducing the pole order of the dominant pole, and apply the construction of [1] only when the dominant pole is simple. This step seems to simplify the construction of [1] in the case when $H_{(s)}(z)$ has a multiple dominant pole.

Again, for the sake of simplicity we will use the notation $H(z)$ instead of $H_{(s)}(z)$, and we will assume that $H(z)$ is primitive with nonnegative impulse response.

Denote in this Step the transfer function corresponding to the "shifted" impulse response sequence $\left(h_{k}, h_{k+1}, \ldots\right)$ by $H_{k}(z)$, i.e.

$$
H_{k}(z):=\sum_{j=1}^{\infty} h_{j+k-1} z^{-j}
$$

(Note that $H(z)=H_{1}(z)$.) First we make the following observation:

If $H_{k}(z)$ has a nonnegative realization in $N$ dimensions (for some $k>1$ ), then so does $H_{k-1}(z)$ in $N+1$ dimensions. (For an easy proof see [7].)

In order to construct a nonnegative realization of $H(z)$ we take the following guidelines: 
We find a positive integer $k_{1}$ so that we can construct a nonnegative realization of $H_{k_{1}}(z)$ in some dimensions $N$, and then use the obsevation above to construct a nonnegative realization of $H(z)$ in dimensions $N+k_{1}-1$. The index $k_{1}$ will be chosen so that

$$
H_{k_{1}}(z)=\left[\sum_{m=1}^{n_{1}} \frac{e_{m}}{(z-1)^{m}}\right]+\left[\frac{d_{1}}{z-1}+\sum_{j=2}^{r} \sum_{i=1}^{n_{j}} \frac{e_{j}^{(i)}}{\left(z-\lambda_{j}\right)^{i}}\right]=: f^{(3)}(z)+f^{(4)}(z)
$$

holds, where $e_{m} \geq 0$ for all $1 \leq m \leq n_{1}$ and the whole impulse response sequence of $f^{(4)}(z)$ is nonnegative. Here $f^{(3)}(z)$ has a trivial positive realization in $n_{1}$ dimensions, and $f^{(4)}(z)$ is primitive with a simple dominant pole and nonnegative impulse response, so that the construction of [1], Theorem 4.1 can be applied. We remark that the index $k_{1}$ is not uniquely determined. In order to minimize the dimension of the realization it is important to determine the optimal value of $k_{1}$. This may be easy to do for a particular transfer function $H(z)$, but a general formula for the optimal value of $k_{1}$ does not seem possible to find. The proof below shows only that such an index $k_{1}$ always exists.

Assume that $n_{1}>1$. Notice that

$$
H_{k}(z)=\sum_{j=1}^{\infty} h_{k+j-1} z^{-j}=z^{k-1} H(z)-\sum_{r=1}^{k-1} h_{r} z^{k-r-1} .
$$

In particular, the formula $H_{2}(z)=z H(z)-h_{1}=(z-1) H(z)+H(z)-h_{1}$ shows that in the partial fraction decomposition of $H_{2}(z)$ the part corresponding to $\lambda_{1}=1$ is given by

$$
\frac{1}{(z-1)^{n_{1}}}+\sum_{j=1}^{n_{1}-1} \frac{c_{1}^{(j)}+c_{1}^{(j+1)}}{(z-1)^{j}}
$$

From this it follows by mathematical induction with respect to $k$ that the partial fraction part corresponding to $\lambda_{1}=1$ in $H_{k}(z)$ is given by

$$
\frac{1}{(z-1)^{n_{1}}}+\sum_{j=1}^{n_{1}-1} \frac{\sum_{i=j}^{n_{1}}\left(\begin{array}{c}
k-1 \\
i-j
\end{array}\right) c_{1}^{(i)}}{(z-1)^{j}}
$$

If $k \geq N_{0}$ (as in Step 3), then $\left(\begin{array}{c}k-1 \\ j\end{array}\right) /\left(\begin{array}{c}k-1 \\ j-1\end{array}\right) \geq n_{1} C$ for all $j=1,2, \ldots, n_{1}-1$. Therefore, we see as in Step 3 that for $k \geq N_{0}$ all the numerators in (4) are not less than 1 . Therefore,

$$
H_{N_{0}}(z)=\left[\sum_{m=2}^{n_{1}} \frac{d_{m}}{(z-1)^{m}}\right]+\left[\frac{d_{1}}{z-1}+\sum_{j=2}^{r} \sum_{i=1}^{n_{j}} \frac{d_{j}^{(i)}}{\left(z-\lambda_{j}\right)^{i}}\right]=: f^{(1)}(z)+f^{(2)}(z) .
$$

where $d_{m} \geq 1$ for all $1 \leq m \leq n_{1}$ (but the first few terms of the impulse response sequence of $f^{(2)}(z)$ may be negative!). We can now apply the estimates of Step 3 to the function $f^{(2)}(z)$ and obtain a number $\tilde{K}_{0}$ such that the impulse response of the function 
$f^{(2)}(z)$ becomes nonnegative if $k \geq \tilde{K}_{0}$. This means that we can take $k_{1}=N_{0}+\tilde{K}_{0}$, and the desired decomposition $H_{k_{1}}(z)=f^{(3)}(z)+f^{(4)}(z)$ holds. Now, the construction of [1] Theorem 4.1 applies to $f^{(4)}(z)$, and we can combine the positive realizations of $f^{(3)}(z)$ and $f^{(4)}(z)$ to get a positive realization of $H_{k_{1}}(z)$. Then we obtain a positive realization of $H(z)$ by applying the observation above.

We remark that an upper bound on the dimension of the positive realization constructed in [1], Theorem 4.1 has not yet been presented. Such dimension estimate is possible to prove, but the proof is fairly long and mathematically involved. It will be presented in a forthcoming publication.

\section{ExAmple}

We illustrate the steps of the algorithm by the following example. Let

$$
H(z)=\frac{1+z-\frac{1}{4} z^{2}}{z^{3}-1}+\frac{1 / 3}{(z-1)^{2}}+\frac{2 / 3}{z-1 / 2}+\frac{2 / 3}{z+1 / 2}-\frac{1}{z+0.9} .
$$

Then $p=3$, and the downsampled functions are

$$
\begin{aligned}
& H_{(0)}(z)=\frac{-1 / 4}{z-1}+\frac{1}{(z-1)^{2}}+\frac{2 / 3}{z-1 / 8}+\frac{2 / 3}{z+1 / 8}-\frac{1}{z+0.729} \\
& H_{(1)}(z)=\frac{4 / 3}{z-1}+\frac{1}{(z-1)^{2}}+\frac{1 / 3}{z-1 / 8}-\frac{1 / 3}{z+1 / 8}+\frac{0.9}{z+0.729} \\
& H_{(2)}(z)=\frac{5 / 3}{z-1}+\frac{1}{(z-1)^{2}}+\frac{1 / 6}{z-1 / 8}+\frac{1 / 6}{z+1 / 8}-\frac{0.81}{z+0.729} .
\end{aligned}
$$

Next we check the nonnegativity of the impulse response of $H(z)$ by checking each $H_{(s)}(z)(s=0,1,2)$. For $s=0$ we have $n_{1}=2, C=5 / 4$ and $N_{0}=4$. Further, following the definitions, we have $N_{1}=3, \rho=0.729, \gamma=1, \eta=1, N^{(1)}=1, C^{(1)}=\sqrt{0.729}$, $K^{(1)}=\frac{\sqrt{0.729}}{3}$, and $\log _{\rho^{1 / 2}} K^{(1)}=7.95$. Therefore it is sufficient to check the first $K_{0}=8$ terms of the impulse response sequence of $H_{(0)}(z)$. Following similar calculations we deduce that it is enough to check the nonnegativity of the first 8 terms of the impulse response sequence of $H_{(1)}(z)$, and the first 7 terms in the case of $H_{(2)}(z)$.

Next, we construct a positive realization for each $H_{(s)}(z)(s=0,1,2)$. In the case of $H_{(0)}(z)$ we apply Step 4 with $k_{1}=1$. The 'shifted' transfer function is given by $H_{2}(z)=\left[\frac{1}{(z-1)^{2}}\right]+\left[\frac{3 / 4}{z-1}+\frac{1 / 12}{z-1 / 8}-\frac{1 / 12}{z+1 / 8}+\frac{0.729}{z+0.729}\right]=f^{(3)}(z)+f^{(4)}(z)$

Now, a positive realization for $f^{(3)}(z)$ and $f^{(4)}(z)$ is given by the triplets $\left(c_{3}, A_{3}, b_{3}\right)$ and $\left(c_{4}, A_{4}, b_{4}\right)$, respectively, where 


$$
\begin{aligned}
& c_{3}=\left(\begin{array}{ll}
1 & 0
\end{array}\right), A_{3}=\left(\begin{array}{ll}
1 & 1 \\
0 & 1
\end{array}\right), b_{3}=\left(\begin{array}{l}
0 \\
1
\end{array}\right), \\
& c_{4}=\left(\begin{array}{llll}
1 & 0 & 1.972 & 0.291412
\end{array}\right), A_{4}=\left(\begin{array}{cccc}
0 & 1 & 0 & 0 \\
1 / 64 & 0 & 0 & 0 \\
0 & 0 & 0 & 0.729 \\
0 & 0 & 1 & 0.271
\end{array}\right), b_{4}=\left(\begin{array}{c}
0 \\
1 / 48 \\
0.75 \\
0
\end{array}\right) .
\end{aligned}
$$

Now we apply the observation of Step 4 (cf. [7]), and give a positive realization of $H_{(0)}(z)$ by

$$
c_{0}=\left(1 / 12, \quad c_{3}, \quad c_{4}\right), A_{0}=\left(\begin{array}{ccc}
0 & 0 & 0 \\
b_{3} & A_{3} & 0 \\
b_{4} & 0 & A_{4}
\end{array}\right), b_{0}=\left(\begin{array}{l}
1 \\
0 \\
0
\end{array}\right)
$$

The cases of $H_{(1)}(z)$ and $H_{(2)}(z)$ are 'trivial' because we can take $k_{1}=0$ in Step 4, and write

$$
\begin{aligned}
& H_{(1)}(z)=\left[\frac{1}{(z-1)^{2}}\right]+\left[\frac{4 / 3}{z-1}+\frac{1 / 3}{z-1 / 8}-\frac{1 / 3}{z+1 / 8}+\frac{0.9}{z+0.729}\right] \\
& H_{(2)}(z)=\left[\frac{1}{(z-1)^{2}}\right]+\left[\frac{5 / 3}{z-1}+\frac{1 / 6}{z-1 / 8}+\frac{1 / 6}{z+1 / 8}-\frac{0.81}{z+0.729}\right] .
\end{aligned}
$$

In both cases positive realizations of dimension 6 can be given as follows:

$$
\begin{gathered}
c_{1}=\left(\begin{array}{llllll}
1 & 0 & 1 & 0 & 1.675 & 0.507925
\end{array}\right), A_{1}=\left(\begin{array}{cccccc}
1 & 1 & 0 & 0 & 0 & 0 \\
0 & 1 & 0 & 0 & 0 & 0 \\
0 & 0 & 0 & 1 & 0 & 0 \\
0 & 0 & 1 / 64 & 0 & 0 & 0 \\
0 & 0 & 0 & 0 & 0 & 0.729 \\
0 & 0 & 0 & 0 & 1 & 0.271
\end{array}\right), b_{1}=\left(\begin{array}{c}
1.354294
\end{array}\right), A_{2}=\left(\begin{array}{cccccc}
1 & 1 & 0 & 0 & 0 & 0 \\
0 & 1 & 0 & 0 & 0 & 0 \\
0 & 0 & 0 & 1 & 0 & 0 \\
0 & 0 & 1 / 64 & 0 & 0 & 0 \\
0 & 0 & 0 & 0 & 0 & 0.729 \\
0 & 0 & 0 & 0 & 1 & 0.271
\end{array}\right), b_{2}=\left(\begin{array}{c}
0 \\
1 \\
0 \\
1 / 12 \\
4 / 3 \\
0
\end{array}\right) \\
c_{2}=\left(\begin{array}{llllll}
1 & 0 & 1 & 0 & 0.514 \\
0 \\
1 \\
1 / 3 \\
0 \\
5 / 3 \\
0
\end{array}\right)
\end{gathered}
$$

A positive realization of the original $H(z)$ is then possible to construct as in [4] or [8]. 


\section{Conclusions}

In this brief we provided a general finite step procedure for checking the nonnegativity of the impulse response sequence of $H(z)$, which answers an open problem raised in [1]. For primitive transfer functions a new method of positive realization was proposed by reducing the pole order of the dominant pole.

\section{REFERENCES}

[1] B.D.O. Anderson, M. Deistler, L. Farina, and L. Benvenuti, "Nonnegative realization of a linear system with nonnegative impulse response," IEEE Trans. Circuits Syst. I, vol. 43, no.2, pp 134-142, Feb. 1996.

$[2]$ L. Benvenuti, L. Farina, and B.D.O. Anderson, "Filtering through a combination of positive filters," IEEE Trans. Circuits Syst. I, vol. 46, no.12, pp 1431-1440, Dec. 1999.

[3] L. Benvenuti and L. Farina, "An example of how positivity may force realizations of 'large' dimensions," Syst. Contr. Lett., vol. 36, no.4, pp 261-266, 1999.

[4] L. Farina, "On the existence of a positive realization," Syst. Contr. Lett., vol. 28, no.4, pp 219-226, 1996.

[5] L. Farina and S. Rinaldi, Positive linear systems: Theory and applications. New York: Wiley, 2000.

[6] K.-H. Foerster, B. Nagy, "Nonnegative realizations of matrix transfer functions," Linear Algebra Appl., vol. 311, no.1-3, pp 107-129, 2000.

[7] C. Hadjicostis, "Bounds on the size of minimal nonnegative realizations for discrete-time LTI systems," Syst. Contr. Lett., vol. 37, no.1, pp 39-43, 1999.

[8] T. Kitano and H. Maeda, "Positive realization of discrete-time system by geometric approach," IEEE Trans. Circuits Syst. I, vol. 45, no.3, pp 308-311, Mar. 1998.

[9] Y. Ohta, H. Maeda, and S. Kodama, "Reachability, observability and realizability of continuous-time positive systems," SIAM J.Control Optim., vol. 22, no.2, pp. 171-180, 1984. 